\title{
TNF- $\alpha$ LOCALIZATION IN THE DENTAL PULP OF ALBINO RATS RECEIVING LONG TERM TREATMENT WITH TRAMADOL HYDROCHLORIDE
}

\author{
Heba Elsaied* and Asmaa Abo Elsoud**
}

\begin{abstract}
Objectives: This investigation estimated the effect of Tramadol hydrochloride on the dental pulp of young male albino rats through histological examination and immune histochemical localization of TNF- $\alpha$.

Materials and Methods: The study sample consisted of 30 young male albino rats which were divided into two groups; the control group (Group I) included 10 rats and the treatment group (Group II) included 20 rats and were given an oral dose consisting of $60 \mathrm{mg} / \mathrm{kg}$ body weight Amadol (tramadol hydrochloride) daily for a period of 4 months.

Results: The light microscopic examination of the dental pulp of the rats received Amadol revealed marked structural degenerative changes in the dental pulp. The immunohistochemical results of the dental pulp of Amadol group showed strongly positive TNF- $\alpha$ staining reactivity of the odontoblasts, fibroblasts, collagen fibers and blood vessels indicating the degenerating process.

Conclusion: long term use of Tramadol has destructive effects on dental pulp so, it must be prescribed and used with precautions.
\end{abstract}

Key words: Tramadol; dental pulp; TNF- $\alpha$.

\section{INTRODUCTION}

The German company Grünenthal $\mathrm{GmbH}$ has launched Tramadol under the name of "Tramal" in 1977 and it was launched later after two decades in the United Kingdom, United States, and Australia ${ }^{1}$. Under several names including Ultram, Zytram, Amadol and others, this medication is sold ${ }^{2}$.
In addition, it is an opioid pain medication which is used for the treatment of pain ranging from moderate to moderately sever ${ }^{3}$. After taking this medication orally, it results in relieving the pain usually within an hour ${ }^{4}$. Further, the medication is used in combination with Paracetamol in order to increase its efficacy in relieving the pain ${ }^{5}$.

* Lecturer, Department of Oral Biology Department, Faculty of Dentistry, Suez Canal University, Egypt

** Lecturer, Department of Pediatric and Preventive Dentistry and Dental Public Health, Faculty of Dentistry, Suez Canal University, Egypt 
The $\mu$-opioid receptor to which it binds in order to achieve its desired results. Furthermore, Tramadol is considered a serotonin-norepinephrine reuptake inhibitor (SNRI) ${ }^{6}$ and it is classified in the class of benzenoid and as the desmetramadol is considered a more powerful opioid, the Tramadol is transformed to it in the body?

When compared to the same dose of Morphine, the power of Tramadol reaches one tenth of that of morphine. Also, it is nearly considered as potent as pethidine and codeine ${ }^{8}$.

When it is used to treat moderately severe pain, it is considered as potent as morphine but in the type of severe pain, it is considered less potent than morphine as its analgesic effects can continue for about 6 hours ${ }^{9}$.

Moreover, the forms of Tramadol include liquids, syrups, drops, and effervescent tablets, powders which are used to be mixed with water, capsules, and tablets including extended release formulations, suppositories and injections ${ }^{10}$.

The drug shouldn't be used in case of pregnancy due to the fact that it could result in some reversible withdrawal effects in the newborn and it shouldn't be used during labor as its onset of action occurs within an hour ${ }^{11}$.

In addition, the list of FDA includes that it shouldn't be used in case of those who are under 12 years old as they could suffer from the slow and difficulty of breathing ${ }^{12}$.

In the USA, it was reported that the addition of Tramadol reached $21.5 \%$ in students of eighth grade and $39.8 \%$ in students of tenth grade ${ }^{13}$.

In Egypt, alcohol and drugs are illegal by religious conviction and law while tobacco is accessible even for adolescents ${ }^{14}$.

During the teen years, young people often suffer from emotional, societal, and somatic catastrophes, they become risky for drug abuse or even addiction ${ }^{15}$.
It was found that in 2014, the prevalence of substances abuse among young adolescent school students in Zagazig was 8.8\% (tramadol $83.3 \%$, cannabis $27.8 \%$, and alcohol $16.7 \%)^{16}$.

Another Egyptian study reported that widespread use of tramadol (32.1\%) among children and adolescents who presented to the Emergency Unit of the Poison Control Center of Ain Shams University Hospitals (PCC-ASU), Egypt, for toxicological assessment .The widespread of tramadol due to its availability without prescription, its easy illegal smuggling and its cheap prices ${ }^{17}$.

Teenagers who abuse drugs may have a higher risk of evolving an addiction when they became adults. Also, can have long-term cognitive and behavioral effects since the teenage brain is still developing ${ }^{17}$.

An increase in the risk of respiratory depression, falls, cognitive impairment and sedation is found in old-aged people and therefore it should be carefully used in patients with liver or kidney failure as it is metabolized in the liver and eliminated by the kidneys. ${ }^{18}$

Tramadol has side effects and the most common ones are nausea, dizziness, dry mouth, indigestion, abdominal pain, vertigo, vomiting, constipation, drowsiness and headache, and it was compared to other types of opioids, it was found that respiratory depression and constipation are less problematic with tramadol ${ }^{19}$.

The overdose of tramadol has risk factors including depression, addiction and seizures and only Naloxone can partially result in reversing the toxic effects of its overuse but it may result in an increase in seizure risk ${ }^{20}$.

The administration of chronic opioid can result in inducing the tolerance of immunity despite the enhancement of immune function by Tramadol, unlike other types of opioids. ${ }^{21}$ 
Long term exposure to tramadol hydrochloride also resulted in devastating histological, immunohistochemical, and ultrastructural changes in the dental pulp which get partially improved after 4 weeks recovery period ${ }^{22}$.

TNF- $\alpha$ increased expression is usually associated with degenerating conditions, as those encountered with long term exposure to tramadol hydrochloride .Also its expression alone can produce inflammation similar to pulpitis and osteitis ${ }^{23}$.

Tramadol does not affect osteoclastic activity and bone resorption and does not cause to change the resulted root resorption either if used in therapeutic doses, but with higher doses can reduce orthodontic tooth movement in rats ${ }^{24}$.

The type of cells which signal protein (cytokine) involved in systemic inflammation include Tumor necrosis factor (TNF) which is also considered one of the cytokines forming the acute phase reaction. Although the Tumor necrosis factor (TNF) is generally produced by activated macrophages, CD4+ lymphocytes, NK cells, neutrophils, mast cells, eosinophils, and neurons also produce $\mathrm{it}^{25}$.

The TNF plays a primary role in regulating the cells of immunity and as an endogenous pyrogen, it can result in inducing fever, apoptotic cell death and inflammation. Moreover, it has the ability to prevent tumorigenesis and viral replication and via IL1 \& IL6 producing cells, it responds to sepsis. Its production dysregulation is included in set of human diseases such as Alzheimer, cancer ${ }^{26}$, major depression ${ }^{27}$, psoriasis $^{28}$ and inflammatory bowel disease (IBD).${ }^{29}$ However, studies linked depression and IBD to the levels of $\mathrm{TNF}^{30}$. In the context of malignancy and parathyroid hormone, the production of TNF could result in secondary hypercalcemia and cancers which are associated to its overproduction ${ }^{26}$.

Tramadol is the most commonly abused substances among teenagers in Egypt ${ }^{16}$, so we should assume that some of them may be drug abusers.

This research was a pioneer and innovative one in investigating the relation between long term abuse and dramatic changes encountered in dental pulp in young adolescents.

The null hypothesis was that tramadol has no effect on dental pulp.

\section{MATERIAL AND METHODS}

A total thirty young male albino rats were used in this study. In order to obtain sufficient sample size, Using alpha $(\alpha)$ level of 0.05 and Beta $(\beta)$ level of 0.05 , the size of effect was 1.24 which means that its power $-95 \%$ and the calculation of sample size was carried out using the version 3.1.9.2 of $\mathrm{G}^{*}$ Power statistical analysis software ${ }^{31}$.

Those rats divided as follows:

Group I (control): consisted of 10 young male albino rats.

Group II (experimental): consisted of 20 young male albino rats, they received Amadol (tramadol hydrochloride) in daily oral dose of $60 \mathrm{mg} / \mathrm{kg}$ body weight ${ }^{32}$ using metallic curved oropharengeal tube for 4 months.

\section{Inclusion criteria ${ }^{33}$ :}

1. Young male albino rats (3 months old).

2. Body weight ranges from 180-200 grams.

3. Good overall health with normal physical examination of rats.

\section{Exclusion criteria:}

1. Unable to meet the inclusion criteria.

2. Current active infection or acute illness of any kind.

3. No medications taking either before or during the experiment.

4. Evidence of malignancy. 
The animals were kept in specially designed cages, 5 animals per cage and labelled according to their group. They fed natural diet and given drinking water adlibitum. They kept under proper condition of temperature and ventilation in the animal house at Faculty of Dentistry Suez Canal University, Ismailia, Egypt.

The percentage of mortality for each group were calculated and recorded, gross examination of head and neck of each animal was carried out through the whole experimental period.

At the end of the experiment the rats of the different groups sacrificed by cervical dislocation. Their mandibles dissected out, cut into two halves, fixed in $10 \%$ neutral buffered formalin, for 4 hours and washed in the buffer for 24 hours at $4^{\circ} \mathrm{c}$ then the specimens decalcified in $10 \%$ ethylene diamine tetra acetic acid (EDTA) PH 7-7.4.

After complete decalcification, jaw specimens washed in the phosphate buffer and prepared for light microscopic examination. They dehydrated in alcohol, cleared in xylene, embedded in paraffin, sectioned and subjected to:

1- Hematoxylin and eosin for histological examination.

2- Masson's trichrome for collagen examination.

3- Immunohistochemical localization of TNF- $\alpha$.

Two thirds of the specimens were processed for histological examination and stained with hematoxylin and eosin (H\&E) and Masson's trichrome stain for structural examination to find out any possible microscopic changes.

The remaining third of the specimens were stained with Immuno-peroxidase avidin/biotin staining for immunohistochemical localization of TNF-alpha IHC Antibody in the dental pulp incubated by primary polyclonal rabbit anti TNFalpha IHC antibody and color developed by DAB.

Sections that were stained with $\mathrm{H} \& \mathrm{E}$, and sections that were stained with Immuno-peroxidase avidin/biotin staining for immunohistochemical localization of TNF-alpha were selected, examined and photographed with Leica DM 1000 light microscopy and Camera using Leica Application suite-LAS software in the centre of excellence of molecular and cellular medicine (CEMCM), Suez Canal University.

The intensity of the immunohistochemical staining results from different groups were histometrically analyzed using Image J (1.46 a, NIH, USA) computer system (1.46 a, NIH, USA).

Images were manually corrected for brightness and contrast. Color thresholding was then performed automatically after which pictures were converted to RGB stack type. Masking of the brown TNF-alpha, immuno-stain was performed by red color where any brown stain of any intensity was considered positive whereas the background grey stain was considered negative. Area fraction was then calculated automatically representing the area percentage of immune positive cells to the total area of the microscopic field.

All data obtained from histomorphometric analysis was statistically described.

\section{Ethics:}

The present study approved from the Research Ethics Committee, Faculty of Dentistry, Suez Canal University code (175/2019).

\section{RESULTS}

Light microscopic results:

\section{A) Heamatoxylin and Eosin results:}

\section{1-Group I: Control group:-}

The pulp tissue was formed of loose vascular connective tissue bound with a layer of specialized cells, the odontoblasts. Odontoblasts were formed of a layer of cells arranged at the periphery of the pulp core and had processes extending to dentin. 
Their crowding gave them pseudo stratified appearance. Active odontoblasts appeared long with intensely basophilic cytoplasm, and large basal nucleus. The pulp core showed a uniform arrangement of its cellular constituent. The fibroblasts constituted the major population of pulpal cells. They were large star shape cells with long processes, and large amount of basophilic cytoplasm. Their nuclei contained one or more prominent nucleoli. Collagen fibers embedded in an amorphous ground substance were seen. (Figure 1, A).

Defensive cells as histiocytes, lymphoid cells, plasma cells and mast cells were sometimes found scattered irregularly throughout the pulp. The pulp also contains vascular and neural elements.

\section{2-Group II: Experimental group:}

The pulp revealed granulation tissue formation, vasodilatation and marked inflammatory cell infiltration (Figure 1 B, C). Separation of the odontoblastic layerfrom thedentin wasfrequently seen(Figure 1,D). Marked pulpal necrosis in the form of massive odontoblastic degeneration, loss of organization of the remaining degenerated cells with marked narrowing of pulp space. Complete absence of pulp tissue could be seen in some sections. (Figure 1, E).

\section{B) Masson's Trichrome stain Results:}

\section{1-Group I: Control group:}

The pulp tissue examination revealed normal distribution of the collagen fibers of the pulp with strongly positive staining reactivity. (Figure $2 \mathrm{~A}$ ).

\section{2-Group II: Experimental group:}

The pulp tissues examination revealed that the collagen fibers was weakly to moderately positive staining with Masson's trichrome stain. (Figure $2 \mathrm{~B}, \mathrm{C}$ ).

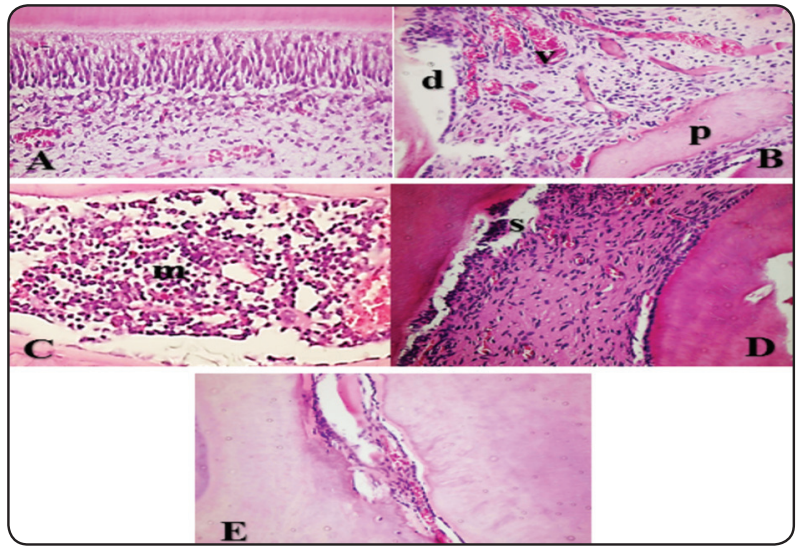

Fig. (1): (A): A photomicrograph of the pulp of the control group showing normal histological structure. (B): The pulp of a rat incisor from the experimental group showing marked vasodilatation, RBCs stagnation \& congestion (v), massive odontoblastic degeneration and loss of organization (d) \& area of dystrophic calcification (false denticle or pulp stone) (p) . (C): Dental pulp of the experimental group showing marked inflammatory cell infiltrate (m). (D): A photomicrograph of the pulp of a rat from the experimental group showing separation of the odontoblastic layer (s). (E): The pulp of from the experimental group showing narrowing of pulp space with generalized pulpal necrosis. (H\&E.orig.mag: A, C, E 200 B, D 400).

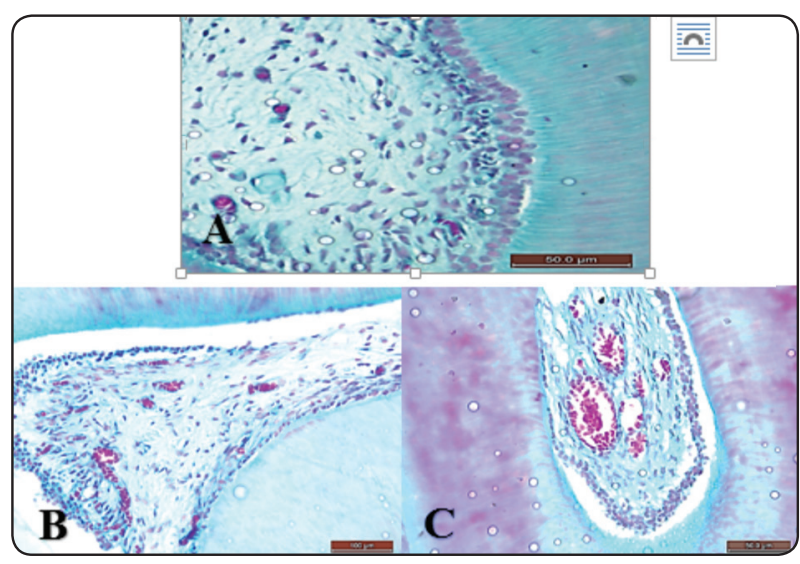

Fig. (2): (A): A photomicrograph of the pulp tissue taken from control group, and stained with Masson's trichrome stain revealed normal distribution of the collagen fibers with strongly positive staining reactivity. $(\mathbf{B}, \mathbf{C})$ : The pulp tissues of group II collagen fibers showed weakly to moderately positive staining with Masson's trichrome stain. (Masson's trichrome, Origin. Mag. B 125, A, C 250). 


\section{II- Immunohistochemical Results:}

\section{1-Group I: Control group:}

By examining the sections which were taken from the dental pulp of rats in this group, it is found that the TNF- $\alpha$ staining reactivity of the cells and fibers was weakly positive. (Fig.3 A, B).

\section{2-Group II: Experimental group:}

By examining the dental pulp of rats, using a light microscope, it is found that TNF- $\alpha$ staining reactivity of the odontoblasts, fibroblasts, collagen fibers and blood vessels was strongly positive indicating that the process of degeneration. (Fig.3 C, D).

Data collected from photomicrographs of sections taken from dental pulp of groups I \& II and stained with TNF- $\alpha$, their stain intensity mean values were calculated using $\mathbf{J}$ image program using Mann-Whitney (U) Test was employed to perform the analysis with Statistical Package for Social Sciences 16.0 for Windows (SPSS Inc., Chicago, IL, USA).

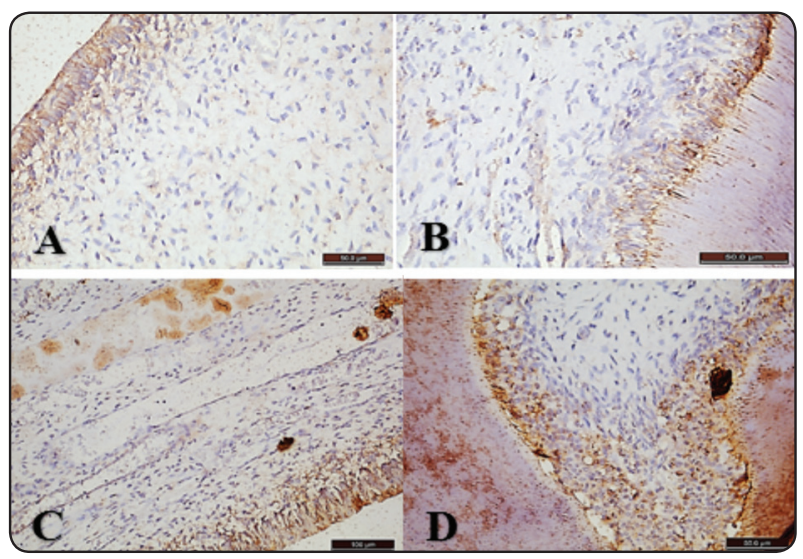

Fig. (3): (A, B): photomicrographs of sections taken from dental pulp of group I showed weakly positive TNF- $\alpha$ staining reactivity of the different cells \& fibers. (TNF- $\alpha$ origin mag. A, B 250). (C, D): photomicrographs of sections taken from dental pulp of group II showed strongly positive TNF- $\alpha$ staining reactivity of the odontoblasts, fibroblasts, collagen fibers and blood vessels.( TNF- $\alpha$ origin mag. C 125, D 250).
Results in table (1) showed highly significant difference between two groups using Mann-Whitney test, the experimental group had the highest value for TNF- $\alpha(198.63 \pm 5.91)$ comparing with control group (170.63 \pm 11.78$)$.

TABLE (1): Showed the mean, standard deviation and range of the TNF- $\alpha$ expression quantification values between control and experimental groups calculated using $\mathrm{J}$ image program.

\begin{tabular}{|c|c|c|c|c|}
\hline & \multirow{2}{*}{$\begin{array}{l}\text { Control } \\
\text { group }\end{array}$} & \multirow{2}{*}{$\begin{array}{l}\text { Experimental } \\
\text { group }\end{array}$} & \multicolumn{2}{|c|}{ Mann-Whitney Test } \\
\hline & & & $\mathrm{U}$ & $\mathrm{p}$ - value \\
\hline Mean & 170.63 & 198.63 & \multirow{3}{*}{-7.8425} & \multirow{3}{*}{$0.00 * *$} \\
\hline $\mathrm{SD}( \pm)$ & 11.78 & 5.91 & & \\
\hline Range & $0-255$ & $0-255$ & & \\
\hline
\end{tabular}

**, means significance between groups at $(P<0.01)$

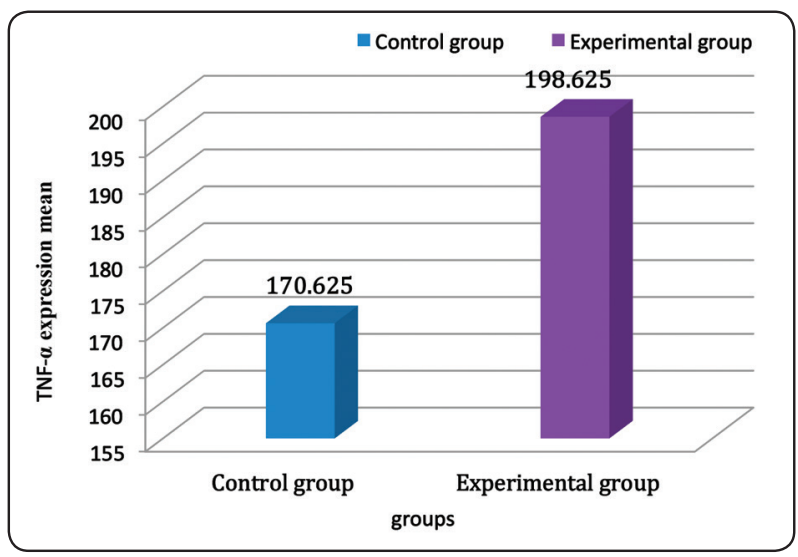

Fig. (4): A bar graph shows the quantification of the TNF- $\alpha$ expression mean values between control and experimental groups. 


\section{DISCUSSION}

Tramadol is a narcotic_like opioid pain reliever that is used to treat moderate to severe pain, even though, it should not be taken if patient suffers from severe breathing problems ${ }^{1}$, stomach or intestines blockage, or current usage of sedatives, tranquilizers or narcotic medication ${ }^{2}$.

When Tramadol is misused, it could result in addiction or even death, particularly in case of children or other people who might use it without prescription. In addition, its use in pregnant women could result in the withdrawal symptoms threatening the lives of newborn. ${ }^{11}$

According to this study, microscopic examination of dental pulp sections obtained from group II (experimental group), revealed granulation tissue formation, vasodilatation and marked inflammatory cell infiltration. Separation of the odontoblastic layer from the dentin was frequently seen marked pulpal necrosis in the form of massive odontoblastic degeneration, loss of organization of the remaining degenerated cells with marked narrowing of pulp space were obvious. Complete absence of pulp tissue could be seen in some sections.

Vasodilatation may be due to the prostaglandin stimulating effect of tramadol.

In terms of flushing and flushing-like symptoms (i.e., diaphoresis and vasodilation) which were found in the administration of Tramadol as related to the dose, these results are considered consistent. However, such symptoms can be reduced using aspirin 30 minutes before tramadol as it inhibits the effect of prostaglandin..$^{34}$

Some reviews ${ }^{35,36}$ have been discussed the antiinflammatory and immunomodulatory properties of opioids, specifically tramadol which appears to have immunostimulatory effects. A temporary increase in airway macrophage numbers in mice that received tramadol was also noted ${ }^{37}$.
Moreover, these findings imply that the effects on chemotaxis are not only drug and dose-specific but may also depend on the anatomic location and the degree of injury as well as the type of inflammatory cell of interest.

These reviews concur with our study which revealed marked inflammatory cell infiltration in group II (experimental group) that received tramadol.

Dilatation of blood vessels and their engorgement with RBCs which results in stasis and decrease in the blood flow are probably the possible causes of hypoxia and ischemia of the tissues that trigger the degenerative effects of tramadol long term use on dental pulp. This finally results in marked pulpal necrosis, odontoblastic degeneration and even complete absence of pulp tissue in some sections.

The TNF $\alpha$ is considered a strong pro-inflammatory agent as it regulates the function of inflammatory cells in several ways. In addition, after trauma or infection, the release of TNF $\alpha$ is fast and it is one of several mediators which are released early in inflamed tissues ${ }^{37}$. The TNF $\alpha$ plays a primary role in the regulation of production of pro-inflammatory cytokine cascade. Accordingly, it is considered the master regulator of production of pro-inflammatory cytokine ${ }^{38}$. Moreover, it results in an increase in the mediators of lipid signal transduction including prostaglandins and platelet activating factor ${ }^{39}$. Considering such roles, it is presented as the master of activation and recruitment of inflammatory cell. Finally, it is suggested to have a fundamental role in developing several chronic inflammatory diseases. ${ }^{40}$

These findings strongly agree with the immunohistochemical localization of TNF- $\alpha$ in the dental pulp group II animals which are subjected to Amadol in a dose of $60 \mathrm{mg} / \mathrm{kg}$ body weight for 4 months that showed strongly positive TNF- $\alpha$ staining reactivity of the odontoblasts, fibroblasts, 
collagen fibers and blood vessels. Also the highly significant difference between two groups at $(\mathrm{P}<0.01)$, as the experimental group had the highest value for TNF- $\alpha$ comparing with control group, indicating the degenerating process in the experimental group.

The results of our investigation also concur with those of another study that held on forty rats receiving a daily oral dose of tramadol $\mathrm{Hcl}$ suspended in saline solution equal to $22.5 \mathrm{mg} / \mathrm{kg} /$ day for nine weeks (therapeutic dose group), and forty rats that received oral dose of tramadol $\mathrm{Hcl}$ suspended in saline solution at doses of 30,60 and $90 \mathrm{mg} / \mathrm{kg} / \mathrm{day}$ on the first, second and third three weeks of the study, respectively for nine weeks (over dose group). The obtained results showed significant increase in serum marker (TNF- $\alpha$ ) levels in both tramadol therapeutic and overdose groups .These results showed that, chronic tramadol administration induced inflammatory stress in rats as well as degenerating endogenous antioxidant defense system mechanisms ${ }^{41}$

These findings are of great importance to be considered in dental treatment of patients who use tramadol in long term condition, also highly beneficial in the treatment of homeless kids, juvenile detentions center and rehabilitation center kids as those are highly suspected to drug addictions as tramadol.

\section{CONCLUSION}

Long term use of Tramadol has destructive effects on dental pulp so, it must be prescribed and used with precautions.

\section{RECOMMENDATION}

Researches on dental pulp of extracted premolars for orthodontic reasons in patients who previously or under long term tramadol

\section{REFERANCES}

1. Leppert W. Tramadol as an analgesic for mild to moderate cancer pain: (PDF). Pharmacological reports 2009; 61: 978-992.

2. Drugs.com. International names for tramadol. Page accessed 2019-4-6.

3. Ramani V, Gauhar A, Khalid B. Tramadol: a valuable treatment for pain in Southeast Asian countries. J Pain Res 2018; 11: 2567-2575.

4. Katz WA. Pharmacology and clinical experience with tramadol in osteoarthritis. Drugs 1996; 52: 39-47.

5. Stephen E, Hartley C, Ioana S, Phil L, Chris F. Analgesic Efficacy of an Acetaminophen/Ibuprofen Fixed-dose Combination in Moderate to Severe Postoperative Dental Pain: A Randomized, Double-blind, Parallel-group, Placebo-controlled Trial. Clinical Therapeutics 2018; 40:1765-1781.

6. Nathan JS, Emily MP, Asha UN. Serotonin Syndrome in the Perioperative Setting. Am J Case Rep 2018; 19: 833-835.

7. Raffa RB, Buschmann H, Christoph T. Mechanistic and functional differentiation of tapentadol and tramadol. Expert Opin Pharmacother 2012; 13: 1437-1449.

8. Grond S, Sablotzki A. Clinical pharmacology of tramadol. Clinical Pharmacokinetics 2004; 43: 879-923.

9. Raffa RB, Buschmann H, Christoph T, Eichenbaum G, Englberger W, Flores CM. Mechanistic and functional differentiation of tapentadol and tramadol. Expert Opin Pharmacother 2012; 13: 1437-149.

10. Rossi S. Australian Medicines Handbook. Adelaide: The Australian Medicines Handbook Unit Trust. ISBN 978-09805790-9-3, 2013

11. Bloor M, Paech MJ, Kaye R. Tramadol in pregnancy and lactation. International Journal of Obstetric Anesthesia 2012; 21: 163-167.

12. Commissioner, Office of the. Press Announcements - FDA statement from Douglas Throckmorton, M.D., deputy center director for regulatory programs, Center for Drug Evaluation and Research, on new warnings about the use of codeine and tramadol in children \& nursing mothers. www.fda.gov. Retrieved 2017-4-21.

13. Fawzy MM. Some medicolegal aspects concerning tramadol abuse: the new Middle East youth plague. An Egyptian overview, Egypt J Forensic Sci 2010; 1:99-102.

14. Bassiony MM, Salah El-Deen MG, Yousef U, Raya Y, AbdelGhani M, El-Gohari H, Atwa S .Adolescent tramadol use and abuse in Egypt. The American Journal of Drug and Alcohol Abuse 2015; 41:206-211. 
15. Neeley WW, Kluemper GT, Hays LR. Psychiatry in orthodontics. Part 2: Substance abuse among adolescents and its relevance to orthodontic practice. Am J Orthod Dentofacial Orthop 2006; 129:185-193.

16. Negm MG, Fouad AA. Prevalence of substance abuse among adolescent school students in Zagazig. Egyptian J of Psychiatry 2014; 35:161-166.

17. Abolmaged S, Kodera A, Okasha T, Gawad T, Rawson R. Tramadol use in Egypt: emergence of a major new public health problem. Can J Addiction Med 2013; 4:5.

18. Amy Wu. Special Considerations for Opioid Use in Elderly Patients with Chronic Pain. US Pharm 2018; 43:26-30.

19. Adams EH, Breiner S, Cicero TJ, Geller A, Inciardi JA, Schnoll SH. A comparison of the abuse liability of tramadol, NSAIDs, and hydrocodone in patients with chronic pain. Journal of Pain and Symptom Management 2006; 31 : 465-476.

20. Keating GM. Tramadol sustained-release capsules. Drugs 2006; 66: 223-230.

21. Al- Hashimi MS, Scott WM, Thompson JP, Lambert DG. Opioids and immune modulation: more questions than answers. BJA: British Journal of Anaesthesia 2013; 111:80-88.

22. Nehad ST. Ultrastructural and Immunohistochemical Investigation on the effect of long term administration of Amadol (Tramadol Hydrochloride) on the Dental Pulp of Albino Rats. Egyptian dental journal 2015; 84: 100- 111.

23. Hall BE, Zhang L, Sun ZJ. Conditional TNF- $\alpha$ Overexpression in the Tooth and Alveolar Bone Results in Painful Pulpitis and Osteitis. J Dent Res 2016; 95: 188-195.

24. Rashidpour M, Ahmad Akhoundi MS, Nik TH, et al. Effect of Tramadol ( $\mu$-opioid receptor agonist) on orthodontic tooth movements in a rat model. J Dent (Tehran). 2012; 9(2):83-9.

25. Locksley RM, Killeen N, Lenardo MJ. The TNF and TNF receptor superfamilies: integrating mammalian biology. Cell 2001; 104: 487-501.

26. Swardfager W, Lanctôt K, Rothenburg L, Wong A, Cappell J, Herrmann N. A meta-analysis of cytokines in Alzheimer's disease. Biol Psychiatry 2010; 68: 930 -941.

27. Dowlati Y, Herrmann N, Swardfager W, Liu H, Sham L, Reim EK. A meta-analysis of cytokines in major depression. Biol Psychiatry 2010; 67: 446-457.

28. Victor FC, Gottlieb AB. TNF-alpha and apoptosis: implications for the pathogenesis and treatment of psoriasis. $\mathrm{J}$ Drugs Dermatol 2002; 1: 264-275.
29. Brynskov J, Foegh P, Pedersen G, Ellervik C, Kirkegaard T, Bingham A. Tumour necrosis factor alpha converting enzyme (TACE) activity in the colonic mucosa of patients with inflammatory bowel disease .Gut 2002; 51: 37-43 .

30. Mikocka-Walus AA, Turnbull DA, Moulding NT, Wilson IG, Andrews JM, Holtmann GJ. Controversies surrounding the comorbidity of depression and anxiety in inflammatory bowel disease patients: a literature review". Inflammatory Bowel Diseases 2007; 13: 225-234.

31. Faul F, Erdfelder E, Lang, AG, Buchner A. G*Power 3: A flexible statistical power analysis program for the social, behavioral, and biomedical sciences. Behavior Research Methods 2007; 39: 175-191.

32. Langley PC, Boswell KA, Benson CJ, Schein, JR. Adverse event profile of tramadol in recent clinical studies of chronic osteoarthritis pain. Current Medical Research and Opinion 2010; 26: 239-251.

33. Sengupta P. The laboratory rat: relating its age with human's .Int J Prev Med 2013; 4:624-630.

34. Ginger L, Wendy S, Alpa N, Helen R. Tramadol-Induced Flushing Managed with Aspirin Premedication. Journal of pain and symptom management 2010; 40: 7-8.

35. Sacerdote P. Opioids and the immune system. Palliat Med 2006; 20: 9-15.

36. Hugunin KM, Fry C, Shuster K, Nemzek JA. Effects of tramadol and burprenorphine on select immunologic factors in a cecal ligation and puncture model. Shock 2010; 34:250-260.

37. Suhana A, Nor A, Jennifer C. The Key Role of TNF-TNFR2 Interactions in the Modulation of Allergic Inflammation: A Review. Front Immunol 2018; 9: 2572.

38. Li Y, Dejin Z, Ren-He X. Critical Role of Tumor Necrosis Factor Signaling in Mesenchymal Stem Cell-Based Therapy for Autoimmune and Inflammatory Diseases. 2018; 9: 1658.

39. Groeger S, Meyle J. Oral Mucosal Epithelial Cells. Front Immunol 2019; 10:208.

40. Clark IA. How TNF was recognized as a key mechanism of disease. Cytokine Growth Factor Rev 2007; 18:335-343.

41. Samy AH, Samir AA, Hany KI .Effect of tramadol drug on some biochemical and immunological parameters in albino male rats: evaluation of possible reversal following its withdrawal. Benha Veterinary Medical Journal 2017; 33: 418-429. 